\title{
Estágio em serviço público de saúde: percepções de estudantes de Odontologia e consonância com propostas curriculares
}

\author{
Carolina da Silva Carvalho*; Kaethlyn Cordeiro Lobachinski*; Muramí Aparecida Graciano de Souza

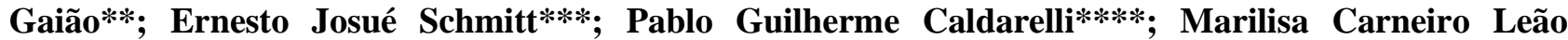 \\ Gabardo****

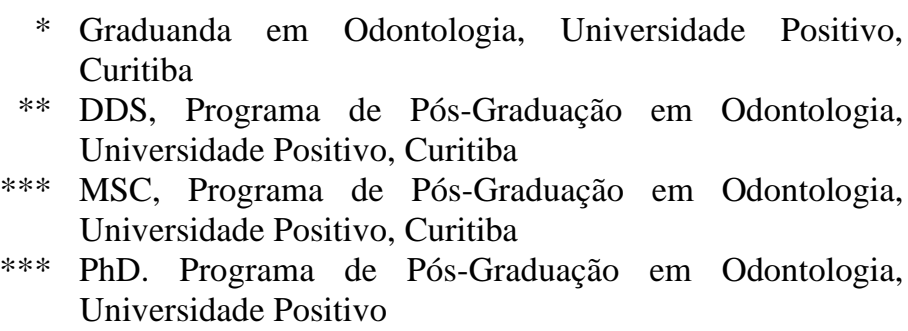

Recebido: 21/11/2019. Aprovado: 22/03/2021.

\begin{abstract}
RESUMO
O estágio supervisionado em serviço público de saúde é o momento no qual o estudante pode colocar em prática toda a sua carga recebida de teoria em diferentes cenários comunitários. A proposta deste estudo foi analisar se a percepção dos estudantes do curso de Odontologia acerca dessa modalidade de estágio está de acordo com os objetivos de aprendizagem da disciplina de Estágio Supervisionado em Serviço de Saúde (ESSS) de uma universidade privada de Curitiba/PR e com as Diretrizes Curriculares Nacionais (DCN). O desenho deste estudo foi o transversal quali-quantitativo. Uma amostra de conveniência contou com 135 estudantes do último ano do curso, de ambos os gêneros e turnos, que haviam concluído a disciplina de ESSS no ano de 2018. Os participantes responderam a um questionário padrão, o qual é entregue ao término do estágio. As perguntas abertas foram analisadas por meio da análise de conteúdo proposta por Bardin e as categorias de análises foram estruturadas de acordo com as competências gerais descritas nas DCN: atenção à saúde, tomada de decisão, comunicação, liderança, administração e gerenciamento e educação permanente. A questão fechada foi analisada, quantitativamente, pela medida de frequências, em SPSS versão 21.0. Os estudantes afirmaram que puderam desenvolver habilidades e competências que corroboram o disposto nos objetivos de aprendizagem da disciplina de ESSS e nas DCN. A classificação do estágio como excelente foi feita por $80,0 \%$ dos pesquisados. Concluiu-se que a percepção dos estudantes acerca do estágio está em consonância com o proposto nos documentos que o norteiam.
\end{abstract}

Descritores: Currículo. Odontologia. Serviços de Saúde. 


\section{INTRODUÇÃO}

O ensino em Odontologia vem, ao longo do tempo, passando por uma série de modificações. Parte-se de um perfil predominantemente voltado para uma formação tecnicista, fragmentada e especializada, fundamentada em um modelo flexneriano de atenção à saúde, até a chegada de um marco na educação superior na área - as Diretrizes Curriculares Nacionais (DCN) para os cursos de graduação em Odontologia ${ }^{1}$. Esse documento surge com a clara proposta de formar indivíduos críticos, reflexivos, humanos, capazes de entender os pacientes como um todo e o adoecer das populações, além de aptos a práticas generalistas. Em sua essência visa potenciais profissionais para o serviço público em saúde ${ }^{1}$, até mesmo porque Sistema Único de Saúde (SUS) tem, dentre suas atribuições, formar recursos para a área da saúde ${ }^{2}$.

Fonseca $(2012)^{3}$ ressalta que as DCN não são apenas um documento em que se registrem termos burocráticos para a adequação dos cursos de graduação em Odontologia no país, mas sim uma representação do avanço da democracia, na qual a saúde é um direito do cidadão assegurado por Lei, somado ao conceito ampliado de saúde e ao que se almeja de um profissional com uma formação sólida e humanizada.

Nas DCN encontram-se descritas as competências gerais e especificas que devem orientar a formação do cirurgião-dentista. Conforme disposto nesse documento orientador, as competências gerais estabelecidas são: atenção à saúde, que incorpora ações de prevenção, promoção, proteção e reabilitação da saúde, em nível individual e coletivo; tomada de decisão, que se refere a tomar decisões com vistas ao uso apropriado, eficaz, com custo-efetividade adequado, capaz de resolver os problemas e elaborar novos conhecimentos; comunicação em que o profissional é acessível, interage com outros profissionais e com o público, transmitindo responsabilidade e confiança; liderança - envolve responsabilidade, habilidade, empatia e compromisso em visar o bem-estar da comunidade; administração e gerenciamento trata-se da capacidade de tomar iniciativas, gerenciar informações e recursos materiais; educação permanente - é a capacidade de aprender continuamente, ter compromisso e responsabilidade com a sua educação ${ }^{1}$.

No rol das competências e habilidades presentes nas DCN do curso de Odontologia é assegurado o desenvolvimento dos estágios curriculares supervisionados, estratégia pedagógica que avança em complexidade ao longo do curso ${ }^{1}$. Nesse contexto, destaca-se o papel fundamental da Associação Brasileira de Ensino Odontológico (ABENO), como uma entidade engajada nas discussões acerca da formação odontológica, com especial atenção aos estágios dos graduandos. Em 2015, uma revisão das diretrizes da ABENO para a execução desses estágios foi publicada ${ }^{4}$. Os autores propuseram que as atividades feitas nos estágios sejam relacionadas diretamente com a realidade do estudante, a fim de que ele tenha uma visão extramuros, um olhar voltado para uma realidade social diferente da sua, de modo a conciliar teoria e prática.

A integração ensino-serviço-comunidade é essencial e o SUS é o cenário ideal onde os estudantes poderão exercer suas práticas sob a perspectiva das DCN junto às comunidades ${ }^{5}$. Estudos indicam que os estágios curriculares na área da saúde permitem que os estudantes reconheçam a realidade de suas profissões em ambientes externos e tenham mais consciência do seu papel junto à sociedade ${ }^{6-8}$.

Nesse processo de construção de experiências participam diversos atores, sejam eles gestores do serviço, preceptores, equipes de trabalho dos equipamentos sociais, docentes e acadêmicos. Há uma necessidade sentida de 
integração entre todos, com propósitos bem definidos, acordos pré-estabelecidos e regras, mas acima de tudo, parceria firmada no sentido de prover o máximo de benefício aos envolvidos.

Estudos acerca da atuação dos preceptores/supervisores em Odontologia identificaram que os mesmos são mediadores da integração dos estudantes com os demais membros da equipe de trabalho e com as rotinas do serviço, além de supervisionar uma série de atividades, sejam elas clínicas, de educação e de promoção da saúde, envoltas em uma troca relevante de experiências ${ }^{9-13}$. Por fim, a análise do desempenho dos acadêmicos é feita por aqueles que acompanharam as práticas in loco ${ }^{10,13}$.

Pesquisas qualitativas têm sido destinadas à compreensão de como ocorre a integração ensinoserviço-comunidade, com diferentes abordagens. Sabe-se que o estágio em serviço de saúde favorece uma formação mais humanística e consistente, onde os acadêmicos aprendem como o serviço de saúde funciona e se tornam capazes de desenvolver atividades fora da esfera meramente clínica ${ }^{14-16}$. No estudo de Luz e Toassi $(2016)^{11}$, estudantes identificaram que o trabalho junto aos preceptores favoreceu a autonomia, a transição do mundo acadêmico para a realidade do mundo do trabalho, uma vez que a aproximação entre esses atores foi relatada como mais coesa, contrariamente ao que ocorre com os docentes. Entretanto, persiste uma lacuna no campo do conhecimento no que se refere à avaliação do estágio em serviço público sob a ótica do acadêmico de Odontologia, apesar da existência de literatura na área ${ }^{15,17}$.

$\mathrm{Na}$ Universidade Positivo (UP), em Curitiba, a matriz curricular segue os pressupostos das $\mathrm{DCN}^{1}$, sendo que os objetivos de aprendizagem da disciplina de Estágio Supervisionado em Serviço de Saúde (ESSS) são: vivenciar diferentes cenários de serviços em saúde, identificar os princípios e as diretrizes do
SUS, reconhecer o processo de trabalho em equipe multidisciplinar, realizar atividades promocionais de saúde junto à comunidade e em equipamentos sociais, executar investigação epidemiológica básica de saúde bucal, compreender o processo de planejamento e programação em saúde bucal coletiva, reconhecer a situação de saúde de indivíduos e comunidades, bem como e as formas de enfrentamento dos problemas de saúde e prestar atendimento odontológico clínico.

Os estudantes do último ano do curso (4. ${ }^{\circ}$ ano do turno na manhã e $5 .^{\circ}$ ano do turno na noite) fazem ESSS no SUS em Unidades de Saúde (US) básicas ou em Unidades da Estratégia de Saúde da Família. Cirurgiões-dentistas, servidores municipais, recebem os acadêmicos nas US e, apesar de não pertencerem ao corpo docente da instituição de ensino, mantêm um relacionamento bastante próximo e auxiliam, inclusive, com sugestões para que as diretrizes da disciplina sejam elaboradas pelos docentes (supervisores indiretos).

Os objetivos de aprendizagem da disciplina de ESSS são: a) vivenciar diferentes cenários de serviços em saúde; b) identificar os princípios e as diretrizes do SUS; c) reconhecer o processo de trabalho em equipe multidisciplinar; d) realizar atividades promocionais de saúde junto à comunidade e em equipamentos sociais; e) executar investigação epidemiológica básica de saúde bucal coletiva; f) reconhecer a situação de saúde de indivíduos e comunidades, bem como e as formas de enfrentamento dos problemas de saúde; e f) prestar atendimento odontológico clínico em US.

Fadel et al. $(2018)^{18}$ relatam que o aprendizado do estudante deve ser amplo, não somente concentrado nas clínicas do curso, mas que que permita a vivência em situações que o levem a desenvolver a capacidade resolvê-las fora do âmbito acadêmico. Assim, a disciplina de 
ESSS da UP se caracteriza por essa intenção. Aos estudantes um "circuito" é proposto, onde cada um deverá identificar, reconhecer e vivenciar o SUS desde a gestão até atividades na "ponta", ou seja, no ambiente clínico. Assim, são oportunizadas visitas aos Distritos Sanitários, participação em reuniões do Conselho Municipal de Saúde e em reuniões multiprofissionais dentro das US, acompanhamento de ações da vigilância sanitária e da vigilância epidemiológica, atividades de educação em saúde, de promoção da saúde, visitas domiciliares e aplicação de ferramentas de trabalho com famílias, como o genograma e o ciclo de vida. Ao término, os acadêmicos promovem um grande evento, com a participação dos envolvidos, e oportunamente são relatadas as experiências vivenciadas. Ainda, os estudantes entregam um relatório de avaliação oficial do estágio, em documento próprio, procedimento padrão na Universidade. Nesse relatório de avaliação, os estudantes têm oportunidade de refletir sobre a vivência dos estágios supervisionados, destacando as contribuições, as potencialidades e desafios da experiência.

Desta forma, buscou-se avaliar se a percepção dos estudantes da disciplina de ESSS do curso de graduação em Odontologia da UP, Curitiba/PR, encontra-se em consonância com os objetivos de aprendizagem propostos e as DCN para os cursos de Odontologia.

\section{METODOLOGIA}

O projeto desta pesquisa foi aprovado pelo Comitê de Ética institucional, sob o parecer 2.636.519 (CAAE: 87994718.0.0000.0093). O desenho de estudo adotado foi o transversal qualiquantitativo. A amostragem de conveniência contou com acadêmicos do último ano ( $4 .^{\circ}$ ano do turno na manhã e $5 .^{\circ}$ ano do turno da noite) do curso de graduação em Odontologia da UP ( $\mathrm{n}=$ 135). Foram incluídos sujeitos de ambos os gêneros e turnos, desde que tivessem cumprido efetivamente a disciplina de ESSS no ano de 2018. Todos assinaram o Termo de Consentimento Livre e Esclarecido.

Os participantes responderam a um questionário autoaplicável, instrumento oficial da UP, entregue ao término do estágio. Esse documento contempla as seguintes informações: dados pessoais, US onde o estágio ocorreu, período de estágio, atividades desenvolvidas durante o estágio, percepção do estudante acerca das atividades terem sido ou não condizentes com a sua formação acadêmica, como se deu a supervisão das atividades no estágio, como eram o local e os recursos disponíveis, de que modo o estágio contribuiu para a sua vida profissional e, por fim, uma avaliação geral do estágio segundo a classificação objetiva em: excelente, muito bom, bom, regular e insuficiente.

Um pesquisador foi responsável pela transcrição das respostas dadas pelos acadêmicos em uma planilha que serviu para o procedimento de análise qualitativa. Para isso, foi utilizada a técnica de análise de conteúdo proposta por Bardin $^{19}$, que incluiu a leitura flutuante dos conceitos apresentados pelos respondentes, criação de categorias de resposta e posterior classificação dos conceitos nas categorias criadas.

Os participantes foram codificados de maneira alfanumérica (E1, E2, E3 ...) garantindo o sigilo da identificação do sujeito respondente.

Foram coletados o Projeto Pedagógico de Curso, os Programas de aprendizagem com as respectivas ementas/objetivos de aprendizagem da disciplina de ESS e as DCN de 2002 ${ }^{1}$. Dessa forma, uma análise documental foi realizada com vistas à discussão dos pressupostos existentes nas DCN e nos objetivos de aprendizagem da disciplina de ESSS. Essa literatura serviu como suporte para a análise conjunta com os dados originais obtidos, o que possibilitou a identificação das categorias de análise. 
Ainda nessa fase qualitativa, para auxiliar a análise dos dados, as respostas dos estudantes foram incluídas no site WordClouds (http://wordclouds.com/) para confecção de uma nuvem de palavras. A nuvem de palavras pode ser compreendida como uma forma de visualização de dados linguísticos, que mostram a frequência com que as palavras aparecem em um dado contexto $^{20}$. Essa técnica consiste na utilização de fontes com diferentes tamanhos, de acordo com a ocorrência das palavras no conteúdo analisado. $\mathrm{O}$ resultado é uma imagem que apresenta um conjunto de palavras, em que a palavra encontrada no centro é a que mais vezes apareceu no conteúdo, e em seu entorno estão as demais palavras de forma decrescente. Ressalta-se que artigos, preposições, locuções adverbiais e palavras que não apresentavam relevância para a análise, foram excluídas para um resultado mais relevante e conciso.

Quanto às questões fechadas, essas foram analisadas quantitativamente pela medida de frequências, com SPSS $\left(\right.$ SPSS $^{\circledR}$ IBM $^{\circledR}$ Corp. Released 2012. IBM SPSS Statistics for Windows, Armonk, NY, EUA), versão 21.0.

\section{RESULTADOS E DISCUSSÃO}

A partir da análise realizada com os dados coletados, observou-se que os estudantes reconheceram que o estágio os colocou em situações em que puderam desenvolver habilidades e competências que corroboram o disposto nos objetivos de aprendizagem da disciplina de ESSS e nas DCN.

Os textos foram organizados de modo que os conteúdos que aparecerem com maior frequência foram agrupados em categorias de análise de acordo com as competências gerais descritas nas DCN para os cursos de Odontologia ${ }^{1}$ : atenção à saúde, tomada de decisão, comunicação, liderança, administração e gerenciamento e educação permanente. No quadro 1 é encontrada uma síntese dos achados da presente pesquisa.

Os estudantes apontam que a participação na disciplina de ESSS é fundamental para a formação, uma vez que experimentam diferentes cenários, reconhecem o sistema público de saúde e aprendem a trabalhar em equipe.

A Atenção à Saúde, definida não somente por aspectos de prevenção, promoção e manutenção da saúde, trata de cuidados assistenciais que devem considerar as diferenças sociais e abranger necessidades individuais e coletivas $^{1,21}$, observou-se que os estudantes puderam praticar essa atenção, o que pode ser exemplificado pela seguinte consideração:

"[...] a cuidar do próximo, se importar mais com os outros e procurar saber o que se passa, em que condições se encontra a pessoa durante as visitas domiciliares e reuniões." (E5)

Dada a diversidade de contextos aos quais os estudantes são expostos, eles conseguem reconhecer que é um direito de todas as pessoas terem acesso à saúde, nesse caso percebido pela frequência dos usuários às US durante o período em que estagiaram. Com isso, os princípios e diretrizes do SUS são atendidos, como a Integralidade refletida no texto abaixo:

"[...] a ser um profissional que se importa mais com a prevenção do que o tratamento restaurador. Ver o paciente como um todo, não somente o problema bucal, não somente tratar o problema, mas sim entender o porquê." (E40)

Durante o processo de ensino-aprendizagem ao longo do curso de graduação em Odontologia da UP, em especial nas disciplinas de Odontologia em Saúde Coletiva, é oportunizada aos estudantes uma visão ampliada do processo saúde-doença, na tentativa de que as práticas tecnicistas e reducionistas sejam superadas. Ênfase é dada à permeabilidade dos aspectos subjetivos envolvidos nesse contexto ${ }^{22}$. 
Estágio em serviço público de saúde: percepções de estudantes de Odontologia e consonância com propostas curriculares

Quadro 1. Síntese dos principais achados em relação às competências gerais

\begin{tabular}{|c|c|}
\hline & ANÁLISE DE CONTEÚDO \\
\hline 选罗 & $\begin{array}{l}\text { "[...] adentrei numa realidade local, além de entender de perto como funciona a atenção primária (básica), os } \\
\text { critérios e o processo de encaminhamento. Há uma integração entre os profissionais [...]" (E22) } \\
\text { "[...] a ser um profissional que se importa mais com a prevenção do que o tratamento restaurador. Ver o paciente } \\
\text { como um todo, não somente o problema bucal, não somente tratar o problema, mas sim entender o porquê." (E40) } \\
\text { "[...] acredito ter desenvolvido muito a minha habilidade de empatia, fazendo com que eu me coloque sempre no } \\
\text { lugar do paciente e busque compreender suas dores, seus problemas, seus sentimentos e frustrações, suas } \\
\text { expectativas, seus sonhos e desejos." (E49) } \\
\text { "[...] como profissional, além de aplicar o teórico, o conhecimento, aprendi muito com as pessoas o verdadeiro } \\
\text { significado de cuidar de vidas." (E32) } \\
\text { "[...] a cuidar do próximo, se importar mais com os outros e procurar saber o que se passa, em que condições se } \\
\text { encontra a pessoa durante as visitas domiciliares e reuniões."(E5) }\end{array}$ \\
\hline 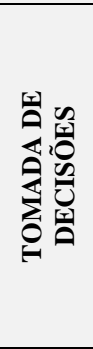 & $\begin{array}{l}\text { "[...] na atividade clínica supervisionada atendi e realizei procedimentos condizentes com o conteúdo abordado na } \\
\text { faculdade [...]. Além disso, também utilizei conteúdo da formação acadêmica para realizar a promoção de saúde nas } \\
\text { escolas municipais." (E135) } \\
\text { "Atendimento aos pacientes com certa autonomia para decisões clínicas. Atendimento com equipe multidisciplinar". } \\
\text { (E24) } \\
\text { "[...] vi muitos casos com diagnósticos que nunca havia visto antes e os solucionei, com a supervisão, mas confiando } \\
\text { em mim mesma. Foram dias de muito aprendizado." (E27) } \\
\text { "Aprendi muito na escolha de qual procedimento é mais conveniente ao paciente, e adaptar a Odontologia para cada } \\
\text { realidade [...]" (E37) }\end{array}$ \\
\hline 至 & $\begin{array}{l}\text { "No estágio na Unidade de Saúde nos deparamos com algumas situaçães que não tivemos na graduação. Auxiliou } \\
\text { em algumas técnicas de manejo do paciente, posicionamento, comunicação com o paciente e como agilizar alguns } \\
\text { procedimentos." (E15) } \\
\text { "O estágio me ajudou muito a melhorar a minha comunicação com pacientes; me auxiliou no manejo, na fala, a como } \\
\text { me portar, e ajudou a convivência em grupos no trabalho." (E36) } \\
\text { "As atividades que não eram diretamente na área da Odontologia, envolviam o relacionamento direto com os } \\
\text { pacientes, ajudando a melhorar a comunicação e conduta frente aos diferentes tipos de casos." (E37) } \\
\text { "[...] habilidade clínica, relacionamento interpessoal, agilidade, domínio de técnica operatório, mais contato com a } \\
\text { real situação bucal dos pacientes [...]" (E30) }\end{array}$ \\
\hline 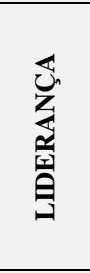 & $\begin{array}{l}\text { "[...] permitiu que assumíssemos pela primeira vez nossa identidade profissional, nos fazendo sentir 'na pele' o } \\
\text { compromisso com os horários, com os pacientes, com a instituição e a Unidade de Saúde que nos confiou atender a } \\
\text { comunidade" (E1) } \\
\text { " [...] contribuiu para decidir rápido os procedimentos, a ter mais controle em situações complicadas e como se } \\
\text { portar como profissional." (E28) } \\
\text { " [...] Aprendi a lidar com diversos tipos de pacientes e situações, a trabalhar com os recursos que a Unidade } \\
\text { possuía." (E5) }\end{array}$ \\
\hline 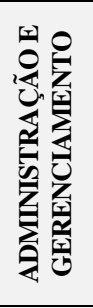 & $\begin{array}{l}\text { "[...] atividades de promoção da saúde, atividade clínica supervisionada, visitas domiciliares com agente de saúde, } \\
\text { gerenciamento de insumos necessários para o atendimento odontológico" (E19) } \\
\text { "[...] observar como funciona a estrutura de uma Unidade de Saúde contribuiu na minha vida profissional } \\
\text { principalmente em como me organizar com os materiais utilizados, aprender sobre os recursos utilizados } \\
\text { diferentemente de um consultório particular, respeitar os horários de atendimentos, conhecer o trabalho das pessoas } \\
\text { que trabalham em um posto de saúde." (E35) } \\
\text { "[...] experiência com vários pacientes por período, exercitando o conhecimento da clínica geral e manejo de } \\
\text { agenda." (E24) }\end{array}$ \\
\hline 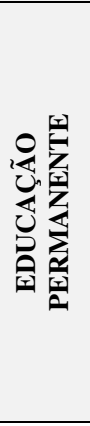 & $\begin{array}{l}\text { "Muito, além da prática das atividades do curso, um aprendizado muito além da Odontologia; conviver com } \\
\text { realidades diferentes, ter uma abordagem para situações inesperadas. Se adaptar ao meio e usar as ferramentas } \\
\text { disponíveis para desenvolver seu melhor trabalho." (E15) } \\
\text { "Permitiu o desenvolvimento de habilidades práticas, socialização de saberes, maior entendimento do processo saúde } \\
\text { - doença, humanização e trabalho multiprofissional." (E9) } \\
\text { " [...] bem acolhido por toda a equipe da Unidade de Saúde, que estava sempre disposta a ajudar e orientar no que } \\
\text { fosse preciso, sempre nos dando liberdade para tirar dúvidas sobre qualquer coisa". (E54) } \\
\text { "O estágio contribuiu de maneira importante para a minha vida profissional. Além do fato de ter tido a oportunidade } \\
\text { de observar, aprender e executar as atividades voltadas para a Odontologia, também pude compartilhar com outros } \\
\text { profissionais da saúde experiências e conhecimentos inclinados não só na saúde do ser humano, mas também ao } \\
\text { cotidiano vivido e voltado à saúde coletiva." (E6) }\end{array}$ \\
\hline
\end{tabular}


Dentre os objetivos da disciplina de ESSS estão a realização de atividades promocionais de saúde junto à comunidade e em equipamentos sociais, e o reconhecimento da situação de saúde dos indivíduos, bem como e as formas de enfrentamento dos problemas de saúde. Essa proposta é confirmada na pesquisa de Bruder et al. $(2017)^{6}$, na qual os estágios em US não são voltados somente para atendimento clínico, mas também para a atenção multiprofissional, a prevenção e a promoção da saúde. Fadel et al. $(2018)^{18}$ apontam a necessidade de valorização do estágio no SUS, dado o benefício da superação das dificuldades a que os estudantes são expostos e também as demandas em saúde pública. Assim, pode-se afirmar que tanto esse item das DCN, quanto os referidos objetivos, corroboram.

A capacidade de tomada de decisão é essencial para qualquer profissional, em especial da área da saúde ${ }^{23}$. Os estudantes perceberam isso, como exemplificado abaixo:

"[...] vi muitos casos com diagnósticos que nunca havia visto antes e os solucionei, com a supervisão, mas confiando em mim mesma. Foram dias de muito aprendizado." (E27)

"Aprendi muito na escolha de qual procedimento é mais conveniente ao paciente, e adaptar a Odontologia para cada realidade [...]" (E37)

Esse ganho em termos de agilidade na prestação do atendimento e de autonomia é favorecido. A execução de casos mais complexos remete à necessidade de ser resgatada a conexão entre as diferentes áreas da Odontologia, com vistas ao paciente ser compreendido como um todo. Segundo Bulgarelli et al. (2014) ${ }^{17}$ o estágio permite aos estudantes olharem para uma realidade extramuros, fazendo com os mesmos se preparem para agir em qualquer situação inesperada fora do âmbito universitário.

Aqui é importante ser ressaltado o papel do preceptor, que cede seu tempo, disponibiliza seu local de trabalho e supervisiona de modo direto todas as atividades executadas. Mesmo não sendo do corpo docente da Instituição de Ensino, é o preceptor que assume o papel de facilitar o aprendizado, guiando os estudantes, fazendo com que haja raciocínio, além de avaliar a conduta dos estudantes e compartilhar suas experiências ${ }^{11,12}$.

A comunicação é uma habilidade pouco explorada em um curso de Odontologia e isso é uma falha, visto que ela melhora a liderança e o relacionamento interpessoal.

"As atividades que não eram diretamente na área da Odontologia, envolviam o relacionamento direto com os pacientes, ajudando a melhorar a comunicação $e$ conduta frente aos diferentes tipos de casos." (E37)

Em Odontologia é necessário que o paciente seja ouvido e que o profissional seja sensível ao que ele relata, compreendendo e respeitando os seus limites ${ }^{24}$. Para se estabelecer uma boa relação entre profissional e paciente, a boa comunicação é necessária e deve ser treinada durante a formação do profissional de Odontologia ${ }^{25}$. Essa melhora foi evidenciada pelo relato:

"O estágio me ajudou muito a melhorar a minha comunicação com pacientes; me auxiliou no manejo, na fala, a como me portar, e ajudou a convivência em grupos no trabalho." (E36)

Há relatos na literatura que a falta de transmissão de informações pelo profissional gera insatisfação ao paciente ${ }^{26}$.

$\mathrm{Na}$ presente pesquisa a categoria temática Liderança ganhou destaque com as devolutivas a seguir:

"[...] permitiu que assumíssemos pela primeira vez nossa identidade profissional, nos fazendo sentir 'na pele' o compromisso com os horários, com os pacientes, com a instituição e a Unidade 
de Saúde que nos confiou atender a comunidade" (E1)

" [...] contribuiu para decidir rápido os procedimentos, a ter mais controle em situações complicadas e como se portar como profissional." (E28)

A liderança é definida como a capacidade de influenciar outrem, ser proativo, e determinar uma direção ${ }^{27}$. Na área odontológica, tornar-se líder é um desafio, essencial para o avanço na profissão ${ }^{28}$. Há indícios na literatura a respeito da falha no sistema de ensino ao se tratar desse quesito $^{29}$. Destaca-se que no trabalho multi/interprofissional em saúde, os sujeitos devem estar aptos para assumirem posições de liderança, tendo em vista a saúde e o bem-estar do indivíduo, família e comunidade. Contudo, tornase importante considerar que a liderança envolve e requer o compromisso, a responsabilidade, a empatia, a habilidade para tomada de decisões, a comunicação e o gerenciamento de forma efetiva e eficaz. Nesse cenário, os estágios supervisionados em serviços de saúde surgem como uma oportunidade ímpar para aproximação e desenvolvimento dessa competência ${ }^{30}$.

Quanto à categoria Administração e Gerenciamento, objetiva-se conseguir organização, eficiência, efetividade e eficácia, mesmo diante dos desafios vivenciados nos serviços públicos de saúde do Brasil. Com base nas respostas dadas, alguns estudantes relataram casos de falta de material, desorganização e problemas com instalações. Contudo, ficou claro que eles compreenderam que o "gestor" está ali para solucionar uma série de problemas, mesmo mediante a escassez de recursos.

"[...] observar como funciona a estrutura de uma Unidade de Saúde contribuiu na minha vida profissional principalmente em como me organizar com os materiais utilizados, aprender sobre os recursos utilizados diferentemente de um consultório particular, respeitar os horários de atendimentos, conhecer o trabalho das pessoas que trabalham em um posto de saúde." (E35)

"Primeiramente nos fez conhecer como funciona o SUS e a Unidade de Saúde em si, fazendo com que pudéssemos agilizar os procedimentos por conta da grande demanda de paciente e agilidade nos atendimentos. Aprendemos também, a fazer nosso melhor com os materiais que temos disponiveis." (E18)

Esses achados corroboram outros relatos encontrados na literatura, que ressaltam que a organização e o compromisso da gestão, somado à participação ativa dos trabalhadores em saúde são essenciais para que os problemas enfrentados pelas US sejam contornados ${ }^{7}$.

Lorenzetti et al. $(2014)^{31}$ afirmam que há uma escassez de profissionais com formação sólida em gestão em saúde, além do fato dessa ser uma área deficiente em termos de educação permanente. Desta forma, este estímulo precoce aos estudantes no estágio, foi alcançado mediante os discursos analisados.

Em relação ao desenvolvimento da competência Educação Permanente, observou-se, pelas avaliações dos estudantes, o reconhecimento da importância de se aprender constantemente e estimular a transformação contínua, pelo acompanhamento de inovações, métodos, vivências dentre outros. Além disso, destaca-se a disponibilidade dos estudantes em se manterem abertos a troca de informações e experiências, por meio de relações pessoais e profissionais, como exemplificado no discurso abaixo:

"O estágio contribuiu de maneira importante para a minha vida profissional. Além do fato de ter tido a oportunidade de observar, aprender $e$ executar as atividades voltadas para a Odontologia, também pude 
compartilhar com outros profissionais da saúde experiências e conhecimentos inclinados não só na saúde do ser humano, mas também ao

cotidiano vivido e voltado à saúde coletiva." (E6)

Morita et al. $(2004)^{5}$ afirmam que a competência Educação Permanente tem seus fundamentos nos pilares da educação, considerando que se baseia na necessidade do aprender constante e contínuo. Os autores destacam, ainda, que a Educação Permanente em Saúde exerce um importante papel de deixar claro que a graduação não é um processo terminal, mas representa apenas o início do aprendizado profissional, que deverá seguir o profissional por toda a vida. Isso ficou evidente na avaliação dos estudantes, quando apontam um aprendizado para "além do curso de Odontologia":

"[...]. além da prática das atividades do curso, um aprendizado muito além da Odontologia; conviver com realidades diferentes, ter uma abordagem para situações inesperadas." (E15)

A reflexão sobre a Educação Permanente como uma competência descrita nas DCN em
Odontologia reforça a necessidade de aproximação das instituições de ensino superior com a sociedade ${ }^{5}$. Nesse sentido, os estágios supervisionados em serviços públicos de saúde podem ser considerados como uma importante estratégia para viabilizar essa aproximação e auxiliar o desenvolvimento dessa competência ${ }^{32}$.

Com base nos dados analisados, a construção da nuvem de palavras contribuiu significativamente para a visualização do que se mostrou mais relevante na avaliação dos estudantes sobre os estágios em serviços de saúde (figura 1). O resultado encontrado corroborou a análise de conteúdo realizada.

De acordo com a figura 1, é possível observar que palavras diretamente relacionadas às competências gerais descritas nas DCN em Odontologia gravitam em torno da palavra "Estágio", com maior destaque para as palavras: "saúde", "comunicação", "autonomia", "gerenciamento", "aprendizado" e "conhecimento". $\mathrm{O}$ resultado assim obtido nessa nuvem de palavras contribuiu, ainda, para se reconhecer a riqueza da experiência do ESSS, oportunizando aos estudantes vivências e contribuições fundamentais para o seu processo de formação.

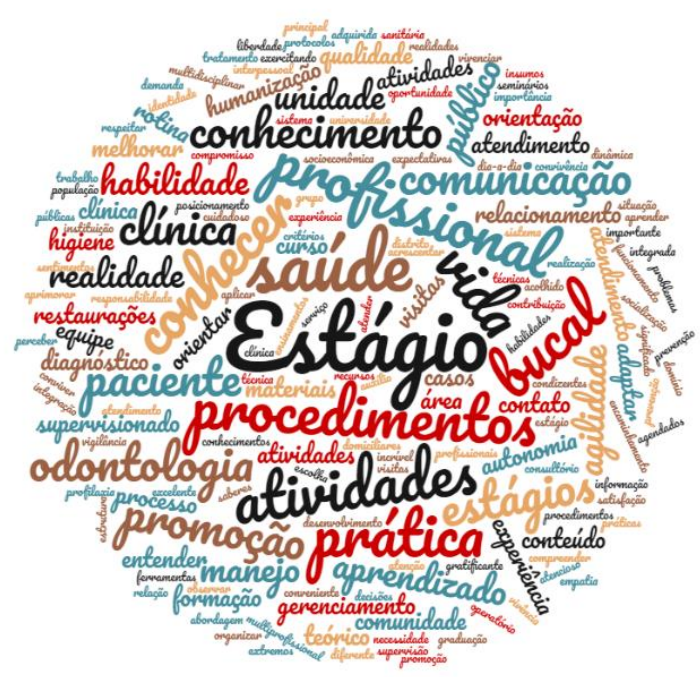

Figura 1. Nuvem de palavras elaborada com base na avaliação dos estudantes sobre a disciplina de ESSS. 
Uma análise a respeito da suficiência das disciplinas teóricas para a prática dos estágios supervisionados em Saúde Coletiva foi realizada por Leite et al. (2018) $)^{33}$. As autoras confirmaram essa suficiência, entretanto há conteúdos que os estudantes indicaram que precisam de maior aprofundamento, como trabalho multiprofissional, gestão de conflitos e ferramentas de trabalho na atenção básica. Essa é uma perspectiva interessante de análise, mas que não foi contemplada na presente pesquisa.

Por fim, os resultados da análise quantitativa revelaram que $80,0 \%$ dos estudantes classificaram o estágio como excelente, $17,8 \%$ como muito bom e 2,2\% como bom. De novo, assim como nos estudos referenciados anteriormente $6,7,15,17,34$, a experiência do estágio foi positiva para os estudantes.

\section{CONCLUSÃO}

Os estudantes de Odontologia da UP, que cursaram a disciplina de ESSS no sistema público, indicaram que as atividades por eles desenvolvidas estão de acordo com o disposto nos objetivos de aprendizagem da disciplina e com as competências gerais descritas nas DCN.

\section{ABSTRACT \\ Internship in public health services: dental students' perceptions and consonance with curricular proposals}

Supervised internships in public health services are the moment when students can put into practice in different community settings all the theory they have learned. The purpose of this study was to analyze whether dental students' perceptions about this type of internship are in accordance with the learning objectives of the Supervised Health Service Internship (SHSI) discipline and the National Curriculum Guidelines (NCG) (Diretrizes Curriculares Nacionais) for Dentistry. This study had a mixed qualitative and quantitative cross-sectional design. A convenience sample of 135 students from the final year of the course, of both genders and from each of the two course shifts, had finished their (SHSI) in 2018. Participants completed a standard questionnaire, which is administered at the end of the internship. The answers to the open-ended questions were analyzed using content analysis as proposed by Bardin and the categories of analysis were structured according to the general competencies described in the NCG: health care, decision making, communication, leadership, administration and management, and continuing education. The answers to the closed-ended question were analyzed quantitatively by measuring frequencies in SPSS version 21.0. The students stated that they were able to develop skills and competencies in keeping with the provisions of the learning objectives of the SHSI discipline and the NCG. The internship was classified as excellent by $80.0 \%$ of respondents. The conclusion was reached that the students' perception about the internship is in line with what is proposed in the documents that guide it.

Descriptors: Curriculum. Dentistry. Health Services.

\section{REFERÊNCIAS}

1. Brasil. Resolução CNE/CES n. 3, de 19 de fevereiro de 2002. Institui as Diretrizes Curriculares Nacionais do Curso de Graduação em Odontologia. Brasília: Diário Oficial da União; 2002.

2. Brasil. Lei $n^{\circ} .8 .080$ de 19 de setembro de 1990. Lei Orgânica da Saúde (LOS). Brasília: Diário Oficial da União; 1990.

3. Fonseca EP. As Diretrizes Curriculares Nacionais e a formação do cirurgião-dentista brasileiro. J Manag Prim Health Care. 2012; 3(2):158-78.

4. Scavuzzi AIF, Gouveia CVD, Carcereri DL, Veeck EB, Ranali J, Costa LJ, Morita MC, Araújo ME. Revisão das Diretrizes da ABENO para a definição do Estágio Supervisionado Curricular nos cursos de Odontologia. Rev ABENO. 2015; 
15(3):109-13.

5. Morita MC, Kriger L. Mudanças nos cursos de Odontologia e sua interação com o SUS. Rev ABENO. 2004; 4(1):17-21.

6. Bruder MV, Lolli LF, Palácios AL, Rocha NB, Veltrini VC, Gasparetto, et al. Estágio supervisionado na odontologia: vivência da promoção da saúde e integração multiprofissional. Rev Bras Promoç Saúde. 2017; 30(2):294-300.

7. Emmi DT, Silva DMC, Barroso RFF. Experiência do ensino integrado ao serviço para formação em Saúde: percepção de alunos e egressos de Odontologia. Interface (Botucatu). 2018; 22(64):223-36.

8. Werneck MAF, Senna MIB, Drumond MM, Lucas SD. Nem tudo é estágio: contribuições para o debate. Ciênc Saúde Coletiva. 2010; 15(1):221-31.

9. Barreto VHL, Monteiro ROS, Magalhães GSG, Almeida RCC, Souza LN. Papel do preceptor da Atenção Primária à Saúde em saúde da formação da graduação e pósgraduação da Universidade Federal de Pernambuco - um termo de referência. Rev Bras Educ Med. 2011; 3(4):578-83.

10. Botti SHO, Rego S. Preceptor, supervisor, tutor e mentor: quais são seus papéis? Rev Bras Educ Med. 2008; 32(3):363-73.

11. Luz GW, Toassi RFC. Percepções sobre o preceptor cirurgião-dentista da Atenção Primária à Saúde no ensino da Odontologia. Rev ABENO. 2016; 16(1):2-12.

12. Pinheiro LCR, Carvalho RB, Viana PFS, Práticas de integração ensino-serviçocomunidade e a formação em Odontologia: possíveis conexões e fluxos no ensino na saúde. Rev ABENO. 2018; 18(4):148-59.

13. Toassi RFC, Davoglio RS, Lemos VMA. Integração ensino-serviço-comunidade: o estágio na atenção básica da graduação em Odontologia. Educ Rev. 2012; 28(4):223-
42.

14. Hood JG. Service learning in dental education: meeting needs and challenges. J Dent Educ. 2009; 73(4):454-63.

15. Mestriner SF, Mesquita LP, Mestriner Júnior W, Bulgarelli AF. Percepções de estudantes de Odontologia sobre a experiência em um estágio não obrigatório no SUS. Rev ABENO. 2017; 17(4):171-82.

16. Toassi RFC, Baumgarten A, Warmling CM, Rossoni E, Rosa AR, Slavutzky SMB. O ensino nos serviços de Atenção Primária à Saúde do Sistema Único de Saúde (SUS) na formação de profissionais de saúde no Brasil. Brasil. Interface (Botucatu). 2013; 17(45):385-92.

17. Bulgarelli AF, Souza KR, Baumgarten A, Souza JM, Rosing CK, Toassi RFC. Formação em saúde com vivência no Sistema Único de Saúde (SUS): percepções de estudantes do curso de Odontologia da Universidade Federal do Rio Grande do Sul (UFRGS), Brasil. Interface (Botucatu). 2014; 18(49):351-62.

18. Fadel CB, Pereira MVS, Moura KC, Martins AS, Pinto MHB. Críticas construtivas de formandos em Odontologia para o repensar do estágio supervisionado no SUS. Rev ABENO. 2018; 19(1):20-32.

19. Bardin L. Análise de conteúdo. São Paulo: Edições 70; 2011.

20. McNaught C, Lam P. Using wordle as a supplementary research tool. The Qualitative Report. 2010; 15(3):630-43.

21. Mendes EV. As redes de atenção à saúde. Ciênc Saúde Coletiva. 2010; 15(5):2297305.

22. Segre M, Ferraz FC. O conceito de saúde. Rev Saúde Pública. 1997; 31(5):538-42.

23. Tanaka OY, Tamaki EM. O papel da avaliação para a tomada de decisão na gestão de serviços de saúde. Ciênc Saúde Coletiva. 
2012; 17(4):821-8.

24. Bark MM, Posanski M, Oliveira KV, Brancher JA, Kriger L, Gabardo MCL. Alterity and empathy: essential virtues for the training of the dentist. Rev ABENO. 2018; 18(2):104-13.

25. Sherman J, Cramer A. Measurement of changes in empathy during dental school. J Dent Educ. 2005; 69(3):338-45.

26. Garbin CAS, Garbin AJI, Dossi AP, Macedo L, Macedo V. O tratamento odontológico: informações transmitidas aos pacientes e motivos de insatisfação. Rev Odontol UNESP. 2008; 37:177-81.

27. Kalenderian E, Skoulas A, Timothé P, Friedland B. Integrating leadership into a practice management curriculum for Dental students. J Dent Educ. 2010; 74(5):464-71.

28. Victoroff KZ, Schneider K, Perry C. Tomorrow's leaders, starting today: A pilot leadership development program for Dental students. J Dent Educ. 2009; 73(3):311-8.

29. D'Assunção FLC, Almeira AC, Kalenderian E. Knowledge of leadership and management: pilot study of student's perceptions of a dentistry course at a university in Brazil. Rev ABENO. 2015; 15(2):28-37.

30. Guerra KJ, Spiri WC. Compreendendo o significado da liderança para o aluno de graduação em enfermagem: uma abordagem fenomenológica. Rev Bras Enferm. 2013; 66(3):399-405.
31. Lorenzetti J, Lanzoni GMM, Assuiti LFC, Pires DEP, Ramos FRS. Gestão em saúde no Brasil: diálogo com gestores públicos e privados. Texto Contexto Enferm. 2014; 23(2):417-25.

32. Silva BS, Caldarelli PG O PET-Saúde em consonância com as diretrizes curriculares nacionais de odontologia no desenvolvimento de competências profissionais: relato de experiência. Rev ABENO. 2013; 13(2):34-41.

33. Leite ANDL, Cassotti E, Gouvêa MV. Análise da suficiência das disciplinas teóricas para a prática dos Estágios Supervisionados em Saúde Coletiva. Diversitates Int J. 2018; 10(2):14-28.

34. Leme PAT, Pereira AC, Meneghim MC, Mialhe FL. Perspectivas de graduandos em odontologia acerca das experiências na atenção básica para sua formação em saúde. Ciênc Saúde Coletiva. 2015; 20(4):1255-65.

\section{Correspondência para:}

Marilisa Carneiro Leão Gabardo

e-mail: marilisagabardo@gmail.com

Rua Prof. Pedro Viriato Parigot de Souza, 5300

81280-330 Curitiba/PR 